\title{
A Novel Method for Investigating the Casimir Effect on Pull-In Instability of Electrostatically Actuated Fully Clamped Rectangular Nano/Microplates
}

\author{
Arman Mohsenzadeh, Masoud Tahani, and Amir R. Askari \\ Department of Mechanical Engineering, Ferdowsi University of Mashhad, P.O. Box 91775-1111, Mashhad, Iran \\ Correspondence should be addressed to Masoud Tahani; mtahani@um.ac.ir
}

Received 30 June 2014; Revised 30 October 2014; Accepted 3 January 2015

Academic Editor: Adriana Szeghalmi

Copyright (C) 2015 Arman Mohsenzadeh et al. This is an open access article distributed under the Creative Commons Attribution License, which permits unrestricted use, distribution, and reproduction in any medium, provided the original work is properly cited.

\begin{abstract}
The objective of the present paper is to represent a novel method to investigate the stable and unstable behaviors of fully clamped rectangular nano/microplates under the effects of electrostatic and Casimir pressures. To this end, the governing partial differential equation of equilibrium is considered and reduced to an algebraic equation using a simple and computationally efficient single degree of freedom (SDOF) model through the Galerkin weighted residual method. The linear and undamped mode-shapes of the plate are used in the Galerkin procedure as the weight function which is obtained by the extended Kantorovich method (EKM). The present findings are compared and validated by available empirical and theoretical results in the literature as well as those obtained by finite element (FE) simulation carried out using COMSOL Multiphysics commercial software and excellent agreements between them are observed.
\end{abstract}

\section{Introduction}

Micron and submicron scale structures are frequently used in different applications such as nano/microelectromechanical systems (N/MEMS) nowadays. Because of their small size, low power consumption, and the reliability of batch fabrications, there are lots of potential applications in engineering $[1,2]$. N/MEMS are also widely spread in biological purposes. Drug delivery, chemical analysis, molecular separation, DNA analysis, and microfluidic systems are some of biological usages of N/MEMS [3, 4].

Some of N/MEMS devices consist of a parallel-plate capacitor as shown in Figure 1. In this figure, the upper nano/microplate is considered as movable electrode which is modelled as a fully clamped nano/microplate and the other one is stationary electrode. Both mechanical and electrical domains play crucial role in N/MEMS. The nano/microplate as mechanical part of the N/MEMS deforms under the electrical loading. This deformation changes the capacitance and also the attracting electrostatic force which acts on the movable electrode. By increasing the input voltage, the microplate deflection may be increased. The induced electrostatic force has an upper limit that overcomes the elastic restoring force and causes the sudden collapse conditions in the nano/microstructure. This unstable behaviour of electrically actuated nano/microstructures is called pull-in instability and the associated voltage and associated displacement are known as pull-in voltage and pull-in displacement, respectively.

Decreasing the dimension of structures from microscale to nanoscale makes the intermolecular surface forces more considerable. The Casimir and the van der Waals attractions are the most important forces which act at the dimensions of N/MEMS [5]. Emission of retarded electromagnetic waves in the separations less than few micrometers makes an intermolecular force, known as the Casimir force [6]. The van der Waals force acts in the shorter distance range compared to the Casimir force which is more considerable in nanoscale [6]. The Casimir force plays a vital role in N/MEMS design [7] inasmuch as neglecting this effect in designing N/MEMS may lead to the collapse condition of the structure without applying any external voltage [8]. Therefore, investigating the Casimir effect on N/MEMS is inescapable. 


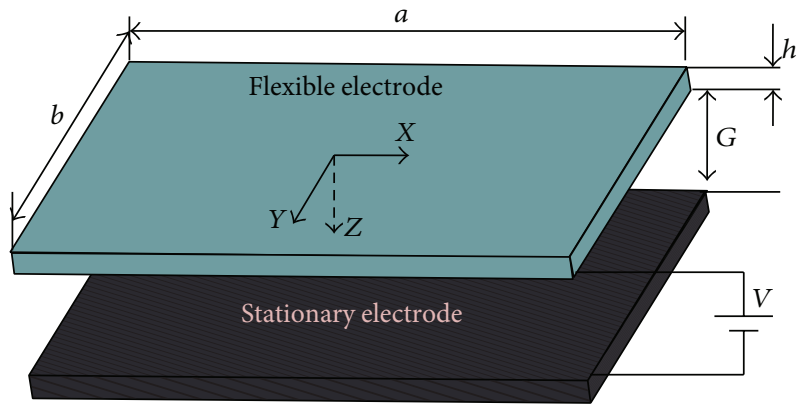

Figure 1: A schematic view of an electrostatically actuated nano/ microplate.

The Casimir effect was mentioned and presented theoretically in 1948 for the first time by the Dutch physicist Hendrik Brugt Gerhard Casimir (1909-2000), who investigates the attraction of two parallel plates in the vacuum [9]. Several researches investigated the role of the Casimir effect on the pull-in phenomenon in N/MEMS [10-13]. Lin and Zhao studied the Casimir effect on the static pull-in and dynamic behaviour of the nanoscale electrostatic actuator using a lumped model [14]. Ramezani et al. investigated pull-in instability of cantilever type NEMS considering both electrostatic and intermolecular surface forces using a distributed parameter model [15]. They used Green's function to transform the nonlinear differential equation of the model into the integral form. Koochi et al. investigated the influence of surface effects including residual surface stress and surface elasticity as well as the effect of Casimir attraction on the pull-in instability of a cantilever nanoactuator [16]. Beni et al. proposed a model to consider the influence of surface effect and molecular forces on pull-in voltage of rotational nano/micromirror using two degrees of freedom model [17]. They also investigated the effect of the Casimir attraction on the torsion/bending coupled pull-in instability of an electrostatic nanoactuator [18]. Farrokhabadi et al. modelled the Casimir force-induced instability in freestanding nanowires with circular crosssection analytically [19]. Zhao et al. suggested a reducedorder model for electrically actuated rectangular microplates. They found the linear mode-shapes numerically using the hierarchical finite element method [20]. Batra et al. presented a review of modelling electrostatically actuated MEMS [21]. They also considered the Casimir force to develop reducedorder models for prestressed clamped rectangular and circular electrostatically actuated microplates [22]. They used their reduced-order models to study the static pull-in instability as well as free vibrations of the plate about its predeformed configuration [22]. They found that using only one mode for transverse deflection in the Galerkin procedure can provide high accurate results [22].

One of the most important issues to model the fully clamped nano/microplates is that the governing equation of the model is strongly nonlinear, so there is no analytical solution for such systems in the literature. In addition, the available numerical solutions suffered from long run time. The main idea of present paper is to represent an accurate solution for such problems which rapidly converges and reduces the time and the cost of the calculation. To this end, the method of extended Kantorovich is employed to extract the accurate mode-shapes of the fully clamped microplate. Using the extracted mode-shapes, the nonlinear governing boundary value equation of equilibrium is reduced to a nonlinear algebraic equation. The resulting nonlinear algebraic equation is also solved through an iterative procedure developed based on Taylor's series expansion. It is shown that the results of present study are in excellent agreement with the finite element simulations carried out using the COMSOL Multiphysics as well as those observed experimentally in the literature.

\section{Problem Formulations}

As shown in Figure 1, a flexible clamped nano/microplate is considered with length $a$, width $b$, thickness $h$, and density $\rho$ under the combined effects of electrostatic and Casimir pressures. The governing equation of motion for deflection of thin nano/microplates can be written as [23]

$$
\begin{gathered}
\rho h \frac{\partial^{2} W}{\partial t^{2}}+D\left(\frac{\partial^{4} W}{\partial X^{4}}+2 \frac{\partial^{4} W}{\partial X^{2} \partial Y^{2}}+\frac{\partial^{4} W}{\partial Y^{4}}\right) \\
=F_{\text {Electrostatic }}(W, V)+F_{\text {Casimir }}(W),
\end{gathered}
$$

where $W$ and $D$ are the deflection and flexural rigidity of the nano/microplate, respectively. Also $F_{\text {Electrostatic }}$ and $F_{\text {Casimir }}$, respectively, denote the distributed electrostatic and the Casimir pressures. These quantities can be defined as [22]

$$
\begin{gathered}
D=\frac{E h^{3}}{12\left(1-v^{2}\right)}, \\
F_{\text {Electrostatic }}=\frac{\varepsilon_{0} \varepsilon_{r} V^{2}}{2(G-W(X, Y))^{2}}, \\
F_{\text {Casimir }}=\frac{\pi^{2} \hbar c}{240(G-W(X, Y))^{4}},
\end{gathered}
$$

where $v$ is Poisson's ratio, $V$ is the applied DC voltage, $G$ is the initial gap between two electrodes, $\varepsilon_{r}$ is the relative permittivity, $\varepsilon_{0}$ is the permittivity of the air, $\hbar=1.055 \times 10^{-34}$ is Planck's constant divided by $2 \pi$, and $c=2.998 \times 10^{8}$ is the speed of light in vacuum. Neglecting the inertia term in (1) leads to the governing static equilibrium equation as follows:

$$
\begin{aligned}
& D\left(\frac{\partial^{4} W}{\partial X^{4}}+2 \frac{\partial^{4} W}{\partial X^{2} \partial Y^{2}}+\frac{\partial^{4} W}{\partial Y^{4}}\right) \\
& =\frac{\varepsilon_{0} V^{2}}{2(G-W(X, Y))^{2}}+\frac{\pi^{2} \hbar c}{240(G-W(X, Y))^{4}} .
\end{aligned}
$$

To nondimensionalize the governing equation of equilibrium, the nondimensional parameters $x=X / a, y=Y / b$, and $w=W / G$ are considered. Substituting these quantities into (5) leads to

$$
\frac{\partial^{4} w}{\partial x^{4}}+2 \alpha^{2} \frac{\partial^{4} w}{\partial x^{2} \partial y^{2}}+\alpha^{4} \frac{\partial^{4} w}{\partial y^{4}}=\frac{\beta}{(1-w)^{2}}+\frac{\gamma}{(1-w)^{4}},
$$


where $\alpha, \beta$, and $\gamma$ are the aspect ratio of the plate $(\alpha=a / b)$, the nondimensional parameter of voltage, and the Casimir force parameter, respectively. $\beta$ and $\gamma$ can also be obtained as

$$
\begin{aligned}
& \beta=\frac{a^{4} \varepsilon_{0} \varepsilon_{r} V^{2}}{2 D G^{3}}, \\
& \gamma=\frac{a^{4} \pi^{2} \hbar c}{240 D G^{5}} .
\end{aligned}
$$

The corresponding nondimensional boundary conditions for fully clamped microplate are

$$
\begin{gathered}
w(x, 0.5)=w(x,-0.5)=0, \\
w(0.5, y)=w(-0.5, y)=0, \\
\left.\frac{\partial w}{\partial x}\right|_{x=0.5,-0.5}=0, \\
\left.\frac{\partial w}{\partial y}\right|_{y=0.5,-0.5}=0 .
\end{gathered}
$$

\section{Solution Procedure}

Due to the high nonlinearity involved in (6), a closedform solution for this equation cannot be found. Hence, an approximate solution will be developed here through the Galerkin weighted residual method. Based on this procedure, the nano/microplate deflection can be expressed as a linear combination of a complete set of linearly independent basis functions [24]. It is noted that these functions must satisfy all kinematic boundary conditions [24]. Therefore, linear and undamped mode-shapes of the undeformed nano/microplate can be used as these basis functions. It is proved that using only the first mode for approximating the transverse deflection in static, dynamic, vibrational, and pull-in analysis of electrically actuated nano/microbeams and plates may be very accurate $[2,4,22,25,26]$. Hence, the deflection of the plate based on one-mode solution can be expressed as

$$
w(x, y)=u \varphi_{11}(x, y),
$$

where $u$ is an unknown parameter determined through the Galerkin procedure and $\varphi_{11}(x, y)$ is the first linear and undamped mode-shape of the undeformed nano/microplate which will be determined in the next section through the extended Kantorovich method (EKM).

According to the Galerkin procedure, (6) is multiplied by $\varphi_{11}(x, y),(10)$ is substituted into the resulting equation, and the outcome is integrated over the dimensionless domain, obtaining

$$
\begin{gathered}
u \int_{-1 / 2}^{1 / 2} \int_{-1 / 2}^{1 / 2}\left(\frac{\partial^{4} \varphi_{11}}{\partial x^{4}}+2 \alpha^{2} \frac{\partial^{4} \varphi_{11}}{\partial x^{2} \partial y^{2}}+\alpha^{4} \frac{\partial^{4} \varphi_{11}}{\partial y^{4}}\right) \varphi_{11} d x d y \\
=\int_{-1 / 2}^{1 / 2} \int_{-1 / 2}^{1 / 2}\left(\frac{\beta \varphi_{11}}{\left(1-u \varphi_{11}\right)^{2}}+\frac{\gamma \varphi_{11}}{\left(1-u \varphi_{11}\right)^{4}}\right) d x d y .
\end{gathered}
$$

Equation (11) is a nonlinear algebraic equation which relates the nondimensional input voltage (i.e., $\beta$ ) and the Casimir parameter (i.e., $\gamma$ ) to the microplate deflection. To solve this equation, the iterative method presented by Tahani and Askari [4] is used and (11) is linearized by expanding its nonlinear terms about an unknown static deflection $u_{0}$ using zeroth-order Tailor's series expansion as

$$
\begin{gathered}
u \int_{-1 / 2}^{1 / 2} \int_{-1 / 2}^{1 / 2}\left(\frac{\partial^{4} \varphi_{11}}{\partial x^{4}}+2 \alpha^{2} \frac{\partial^{4} \varphi_{11}}{\partial x^{2} \partial y^{2}}+\alpha^{4} \frac{\partial^{4} \varphi_{11}}{\partial y^{4}}\right) \varphi_{11} d x d y \\
\quad=\int_{-1 / 2}^{1 / 2} \int_{-1 / 2}^{1 / 2}\left(\frac{\beta \varphi_{11}}{\left(1-u_{0} \varphi_{11}\right)^{2}}+\frac{\gamma \varphi_{11}}{\left(1-u_{0} \varphi_{11}\right)^{4}}\right) d x d y
\end{gathered}
$$

Therefore, the solution takes the form

$$
\begin{gathered}
u=\left(\int_{-1 / 2}^{1 / 2} \int_{-1 / 2}^{1 / 2}\left(\frac{\beta \varphi_{11}}{\left(1-u_{0} \varphi_{11}\right)^{2}}+\frac{\gamma \varphi_{11}}{\left(1-u_{0} \varphi_{11}\right)^{4}}\right) d x d y\right) \\
\cdot\left(\int _ { - 1 / 2 } ^ { 1 / 2 } \int _ { - 1 / 2 } ^ { 1 / 2 } \left(\frac{\partial^{4} \varphi_{11}}{\partial x^{4}}+2 \alpha^{2} \frac{\partial^{4} \varphi_{11}}{\partial x^{2} \partial y^{2}}\right.\right. \\
\left.\left.+\alpha^{4} \frac{\partial^{4} \varphi_{11}}{\partial y^{4}}\right) \varphi_{11} d x d y\right)^{-1} .
\end{gathered}
$$

Hence, the solution can be found through the following iterative formula:

$$
\begin{gathered}
u_{N+1} \\
=\left(\int_{-1 / 2}^{1 / 2} \int_{-1 / 2}^{1 / 2}\left(\frac{\beta \varphi_{11}}{\left(1-u_{N} \varphi_{11}\right)^{2}}+\frac{\gamma \varphi_{11}}{\left(1-u_{N} \varphi_{11}\right)^{4}}\right) d x d y\right) \\
\cdot\left(\int _ { - 1 / 2 } ^ { 1 / 2 } \int _ { - 1 / 2 } ^ { 1 / 2 } \left(\frac{\partial^{4} \varphi_{11}}{\partial x^{4}}+2 \alpha^{2} \frac{\partial^{4} \varphi_{11}}{\partial x^{2} \partial y^{2}}\right.\right. \\
\left.\left.+\alpha^{4} \frac{\partial^{4} \varphi_{11}}{\partial y^{4}}\right) \varphi_{11} d x d y\right)^{-1} .
\end{gathered}
$$

At the first step, the value of $u_{N}$ is set to zero and the iterative procedure is continued till the convergence is achieved or pull-in happened. The convergence criterion is defined as

$$
\left|\frac{\left(u_{N+1}-u_{N}\right)}{u_{N}}\right| \leq 10^{-6},
$$

and the pull-in will happen if

$$
w_{\text {mid-point }}=\varphi_{11}(0,0) u_{N} \geq 1 .
$$

It is to be noted that although we use $w_{\text {mid-point }} \geq 1$ for pullin condition in our solution, this instability can be observed when the slope of deflection-voltage graph reaches infinity [4]. 


\section{Application of the EKM for Obtaining Linear and Undamped Mode-Shapes}

The governing eigenvalue equation for the free vibrations of a microplate can easily be obtained as

$$
\frac{\partial^{4} \varphi_{\mathrm{mn}}}{\partial x^{4}}+2 \alpha^{2} \frac{\partial^{4} \varphi_{\mathrm{mn}}}{\partial x^{2} \partial y^{2}}+\alpha^{4} \frac{\partial^{4} \varphi_{\mathrm{mn}}}{\partial y^{4}}-\omega_{\mathrm{mn}}^{2} \varphi_{\mathrm{mn}}=0
$$

where $\omega_{\mathrm{mn}}$ represents the natural frequency of the microplate. According to the general procedure of the EKM, the microplate mode-shape should be considered as a multiplication of single term separable functions as

$$
\varphi_{\mathrm{mn}}(x, y)=f(x) g(y),
$$

where $f(x)$ and $g(y)$ are unknown functions in terms of $x$ and $y$ to be determined. The dimensionless boundary conditions presented in (9a), (9b), (9c), and (9d) in terms of the separable functions can be written as

$$
\begin{aligned}
& f\left(\frac{-1}{2}\right)=f\left(\frac{1}{2}\right)=\left.\frac{d f}{d x}\right|_{x=-1 / 2}=\left.\frac{d f}{d x}\right|_{x=1 / 2}=0, \\
& g\left(\frac{-1}{2}\right)=g\left(\frac{1}{2}\right)=\left.\frac{d g}{d x}\right|_{x=-1 / 2}=\left.\frac{d g}{d x}\right|_{x=1 / 2}=0 .
\end{aligned}
$$

Based on the general procedure of the Galerkin weighted residual method (GWRM) [24], the variational form of (17) can be obtained as

$$
\begin{aligned}
\int_{-1 / 2}^{1 / 2} \int_{-1 / 2}^{1 / 2}[ & \frac{\partial^{4} \varphi_{\mathrm{mn}}}{\partial x^{4}}+2 \alpha^{2} \frac{\partial^{4} \varphi_{\mathrm{mn}}}{\partial x^{2} \partial y^{2}} \\
& \left.+\alpha^{4} \frac{\partial^{4} \varphi_{\mathrm{mn}}}{\partial y^{4}}-\omega_{\mathrm{mn}}^{2} \varphi_{\mathrm{mn}}\right] \delta \varphi_{\mathrm{mn}} d x d y=0
\end{aligned}
$$

According to the iterative nature of the EKM, at the first step, it is assumed that $g$ is a prescribed known function. Hence, the variation of $\varphi_{\mathrm{mn}}$ would be just because of the variation of the unknown function $f(x)$. Substituting (18) into (20) leads to

$$
\begin{aligned}
\int_{-1 / 2}^{1 / 2}\left\{\int_{-1 / 2}^{1 / 2}[\right. & g \frac{d^{4} f}{d x^{4}}+2 \alpha^{2} \frac{d^{2} f}{d x^{2}} \frac{d^{2} g}{d y^{2}} \\
& \left.\left.+\alpha^{4} f \frac{d^{4} g}{d y^{4}}-\omega_{\mathrm{mn}}^{2} f g\right] g d y\right\} \delta f d x=0
\end{aligned}
$$

Using the fact that $\delta f$ is an arbitrary value except at the boundaries and employing the fundamental lemma of variational calculus, we obtain

$$
\begin{aligned}
& \left(\int_{-1 / 2}^{1 / 2} g^{2} d y\right) \frac{d^{4} f}{d x^{4}}+\left(2 \alpha^{2} \int_{-1 / 2}^{1 / 2} g \frac{d^{2} g}{d y^{2}} d y\right) \frac{d^{2} f}{d x^{2}} \\
& +\left(\alpha^{4} \int_{-1 / 2}^{1 / 2} g \frac{d^{4} g}{d y^{4}} d y-\omega_{\mathrm{mn}}^{2} \int_{-1 / 2}^{1 / 2} g^{2} d y\right) f=0 .
\end{aligned}
$$

Next, using the integration by parts, one can write

$$
\frac{d^{4} f}{d x^{4}}-I_{1} \frac{d^{2} f}{d x^{2}}+\left(I_{2}-\omega_{\mathrm{mn}}^{2}\right) f=0
$$

where

$$
\begin{aligned}
& I_{1}=\frac{2 \alpha^{2} \int_{-1 / 2}^{1 / 2}(d g / d y)^{2} d y}{\int_{-1 / 2}^{1 / 2} g^{2} d y}, \\
& I_{2}=\frac{\alpha^{4} \int_{-1 / 2}^{1 / 2}\left(d^{2} g / d y^{2}\right)^{2} d y}{\int_{-1 / 2}^{1 / 2} g^{2} d y} .
\end{aligned}
$$

At the next step, using the prescribed known function $f$ obtained from (23), the unknown function $g$ can be determined through the following equation:

$$
\frac{d^{4} g}{d y^{4}}-I_{1}^{\prime} \frac{d^{2} g}{d y^{2}}+\left(I_{2}^{\prime}-\omega_{\mathrm{mn}}^{2}\right) g=0
$$

where

$$
\begin{aligned}
& I_{1}^{\prime}=\frac{2 \int_{-1 / 2}^{1 / 2}(d f / d x)^{2} d x}{\alpha^{2} \int_{-1 / 2}^{1 / 2} f^{2} d x}, \\
& I_{2}^{\prime}=\frac{\int_{-1 / 2}^{1 / 2}\left(d^{2} f / d x^{2}\right)^{2} d x}{\alpha^{4} \int_{-1 / 2}^{1 / 2} f^{2} d x} .
\end{aligned}
$$

By choosing an initial guess function $g(y)=\left(4 y^{2}-1\right)^{2}$, the solution of (23) takes the form

$$
\begin{aligned}
f(x)= & C_{1} \sinh (\vartheta x)+C_{2} \cosh (\vartheta x) \\
& +C_{3} \sin (v x)+C_{4} \cos (v x),
\end{aligned}
$$

where

$$
\begin{gathered}
\vartheta=\sqrt{\frac{I_{1}}{2}+\sqrt{\omega_{\mathrm{mn}}^{2}+\left(\frac{I_{1}}{2}\right)^{2}-I_{2}}} \\
v=\sqrt{-\frac{I_{1}}{2}+\sqrt{\omega_{\mathrm{mn}}^{2}+\left(\frac{I_{1}}{2}\right)^{2}-I_{2}}} .
\end{gathered}
$$

Imposing the boundary conditions presented in (19a) yields the following eigenvalue, eigenfunction equations:

$$
\begin{aligned}
& C_{1} \sinh \left(\frac{\vartheta}{2}\right)-C_{2} \cosh \left(\frac{\vartheta}{2}\right) \\
& +C_{3} \sin \left(\frac{v}{2}\right)-C_{4} \cos \left(\frac{v}{2}\right)=0,
\end{aligned}
$$




$$
\begin{gathered}
C_{1} \sinh \left(\frac{\vartheta}{2}\right)+C_{2} \cosh \left(\frac{\vartheta}{2}\right) \\
+C_{3} \sin \left(\frac{v}{2}\right)+C_{4} \cos \left(\frac{v}{2}\right)=0, \\
\vartheta C_{1} \cosh \left(\frac{\vartheta}{2}\right)-\vartheta C_{2} \sinh \left(\frac{\vartheta}{2}\right) \\
+v C_{3} \cos \left(\frac{v}{2}\right)+v C_{4} \sin \left(\frac{v}{2}\right)=0, \\
\vartheta C_{1} \cosh \left(\frac{\vartheta}{2}\right)+\vartheta C_{2} \sinh \left(\frac{\vartheta}{2}\right) \\
+v C_{3} \cos \left(\frac{v}{2}\right)-v C_{4} \sin \left(\frac{v}{2}\right)=0 .
\end{gathered}
$$

This system of equations can be simplified to

$$
\begin{gathered}
C_{2} \cosh \left(\frac{\vartheta}{2}\right)+C_{4} \cos \left(\frac{v}{2}\right)=0, \\
\vartheta C_{2} \sinh \left(\frac{\vartheta}{2}\right)-v C_{4} \sin \left(\frac{v}{2}\right)=0, \\
C_{1} \sinh \left(\frac{\vartheta}{2}\right)+C_{3} \sin \left(\frac{v}{2}\right)=0, \\
\vartheta C_{1} \cosh \left(\frac{\vartheta}{2}\right)+v C_{3} \cos \left(\frac{v}{2}\right)=0 .
\end{gathered}
$$

It is worth noting that (30a), (30b), (31a), and (31b) are related to the symmetric and antisymmetric mode-shapes, respectively. Equating the determinant of the coefficients of these two systems of equations would lead to the following frequency equations for symmetric and antisymmetric modes, respectively:

$$
\begin{aligned}
& v \tan \left(\frac{v}{2}\right)=-\vartheta \tanh \left(\frac{\vartheta}{2}\right), \\
& \vartheta \tan \left(\frac{v}{2}\right)=v \tanh \left(\frac{\vartheta}{2}\right) .
\end{aligned}
$$

After finding the values of $\vartheta$ and $v$, by normalizing the mode-shapes (i.e., setting $C_{4}=1$ for symmetric modes and $C_{3}=1$ for antisymmetric modes), the value of $C_{2}$ and $C_{1}$ can be obtained using (30a) and (31a), respectively. Next, by continuing the iterative procedure, one can utilize the solution of (23) (i.e., (27)) as a prescribed function and find the unknown function $g(y)$ using (25). The procedure should be continued till the convergence occurs.

\section{Finite Element Analysis}

In this section, a fully clamped nano/microplate is modelled using commercial FE software COMSOL Multiphysics. The model is designed to investigate pull-in instability of a nano/microplate under the effect of electrostatic field induced by applying DC voltage. Geometrical and material properties
TABLE 1: Specifications of the system for FE simulation.

\begin{tabular}{lc}
\hline Specification & Value \\
\hline Width $(\mu \mathrm{m})$ & 250 \\
Length $(\mu \mathrm{m})$ & 250 \\
Thickness $(\mu \mathrm{m})$ & 3 \\
Young's modulus $(\mathrm{GPa})$ & 169 \\
Poisson's ratio & 0.28 \\
Density $\left(\mathrm{Kg} / \mathrm{m}^{3}\right)$ & 2331 \\
Initial gap $(\mu \mathrm{m})$ & 1 \\
Dielectric of air $(\mathrm{F} / \mathrm{m})$ & $8.85 \times 10^{-12}$ \\
\hline
\end{tabular}

of the model are also given in Table 1. To find pull-in voltage using COMSOL Multiphysics software, for the first step, the stationary problem should be solved [27]. To do so, a 3D tetrahedral free mesh is employed. Also the electrical field is applied by assigning zero and stationary electric potentials to the movable and fixed electrodes, respectively. The stationary electric potential is also set to vary from $0 \mathrm{~V}$ to the value in which pull-in instability occurs. Pull-in voltage would be found by solving an inverse problem in COMSOL [27]. In this procedure, COMSOL changes the applied voltage to satisfy the equation of the inverse problem. In this study, the FE results obtained by COMSOL are utilized to validate the findings of the present approach.

In order to apply the Casimir effect to the FE model, COMSOL functions are employed. The Casimir force is applied to the bottom surface of the flexible electrode (i.e., the microplate) as a distributed variable boundary load per unit area and its value is obtained through (4). It is to be noted that the Casimir force is a function of deflection, so it is varying in a range that should be set by the user based on the usual range of the pull-in displacements.

\section{Results and Discussions}

6.1. Comparison and Validation. To find the number of iteration which must be carried out in the EKM, a convergence study is performed in Table 2 for square microplate first mode-shape parameters as well as its fundamental natural frequency. From Table 2, it is apparent that the convergence is so rapid and that in all cases it occurs after the second iteration. It should be noted that the calculated present fundamental natural frequency of the system is in an excellent agreement with $\omega_{11}=36.1087$ obtained by Arenas [28]. To illustrate the accuracy of the present analysis, the first modeshape of the microplate is also depicted in Figure 2.

To validate the accuracy of present pull-in results, variation of dimensionless mid-point deflection versus the nondimensional applied voltage for a square microplate without the effect of Casimir force is depicted in Figure 3. The results are compared and validated by available experimental and other existing results in the literature $[20,29]$. As one can observe from this figure, the present findings are in excellent agreement with those reported in previous studies [20, 29]. As Figure 3 shows, the slope of mid-point deflection-voltage graph reaches infinity when the input voltage exceeds a 
TABLE 2: Convergence study for parameters of the first mode-shape as well as dimensionless fundamental natural frequencies of a square microplate.

\begin{tabular}{|c|c|c|c|c|c|c|c|c|}
\hline$i$ & $\left.\vartheta\right|_{i, i-1}$ & $\left.\vartheta\right|_{i, i}$ & $\left.v\right|_{i, i-1}$ & $\left.v\right|_{i, i}$ & $\left.C_{2}\right|_{i, i-1}$ & $\left.C_{2}\right|_{i, i}$ & $\left.\omega_{11}\right|_{i, i-1}$ & $\left.\omega_{11}\right|_{i, i}$ \\
\hline 1 & - & 6.52637011 & - & 4.31201887 & - & 0.04221306 & 35.99948133 & 35.99948133 \\
\hline 2 & 6.52610255 & 6.52610827 & 4.31206780 & 4.31206675 & 0.04222025 & 0.04222010 & 35.99896276 & 35.99896276 \\
\hline 3 & 6.52610815 & 6.52610815 & 4.31206678 & 4.31206678 & 0.04222010 & 0.04222010 & 35.99896276 & 35.99896276 \\
\hline 4 & 6.52610815 & 6.52610815 & 4.31206678 & 4.31206678 & 0.04222010 & 0.04222010 & 35.99896276 & 35.99896276 \\
\hline
\end{tabular}

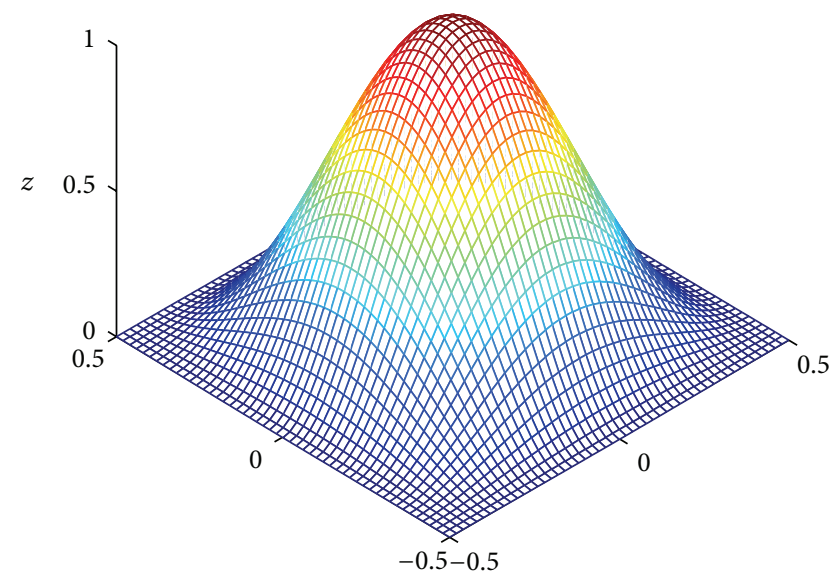

FIGURE 2: The first mode-shape of a fully clamped square microplate.

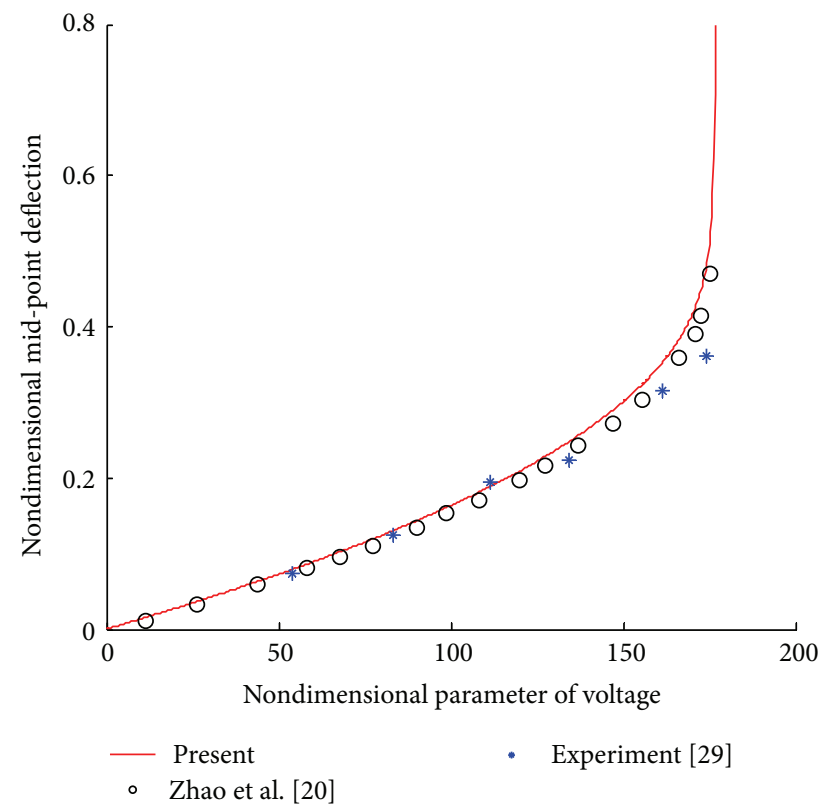

Figure 3: Comparison between present findings and available empirical [29] and numerical [20] results for a square microplate.

critical value called pull-in voltage. It is noteworthy that this fact was used by some researchers to determine the pull-in condition in electrically actuate microbeams $[14,30]$.

To emphasize the accuracy of the present analysis, FE simulation is also employed in the present paper. Figure 4



FIGURE 4: Deformed configuration of the system with properties given in Table 1 at pull-in condition.

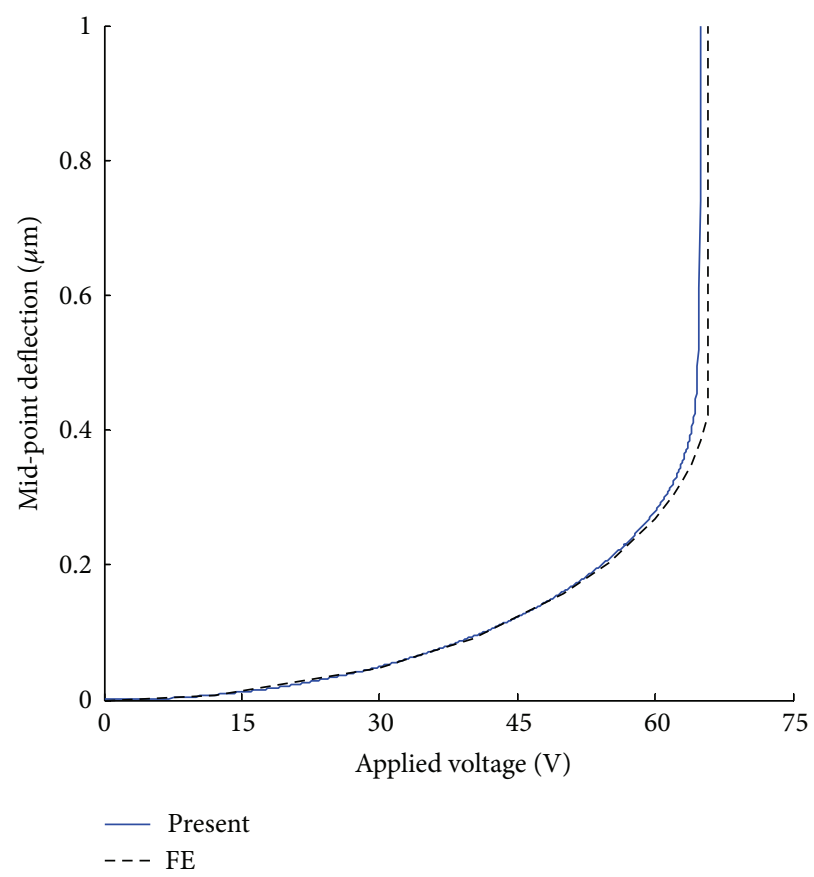

FIGURE 5: Comparison between present findings and FE results for a square microplate with properties listed in Table 1.

shows the deformed configuration of a microsystem modelled in COMSOL Multiphysics software. It should be noted that the properties of the model were also presented in Table 1. As it is seen from Figure 4, the pull-in displacement (the maximum displacement occurred just before bechancing 


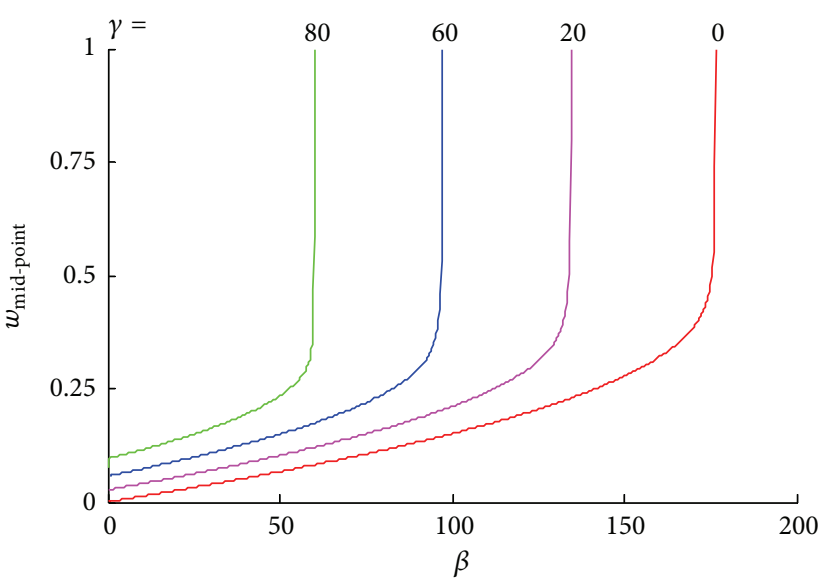

(a)

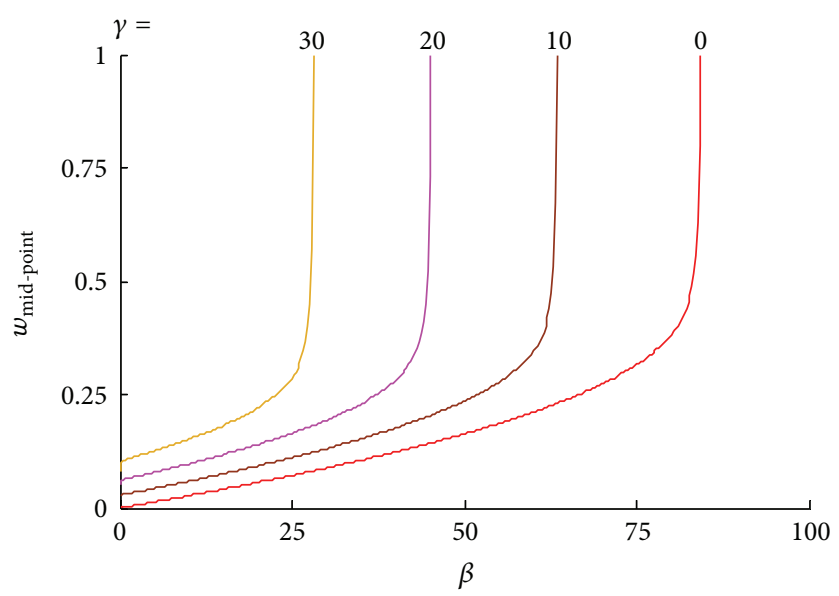

(c)

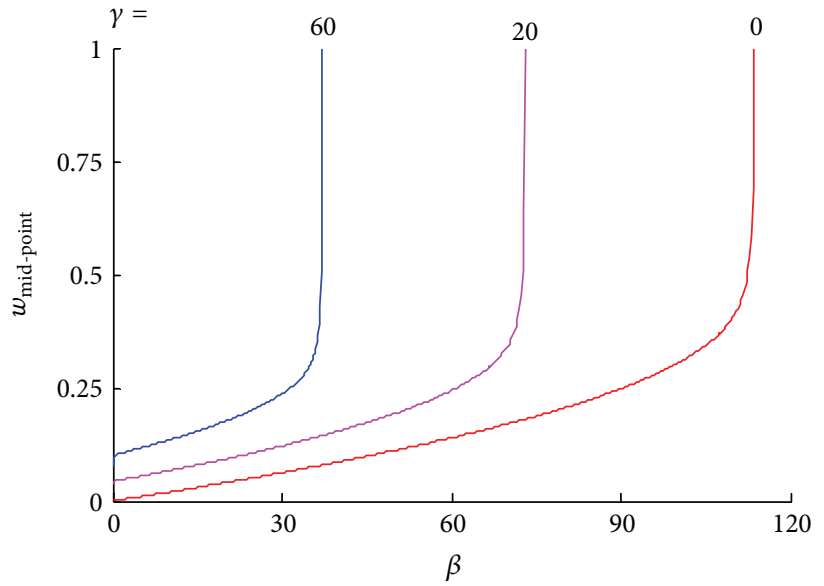

(b)

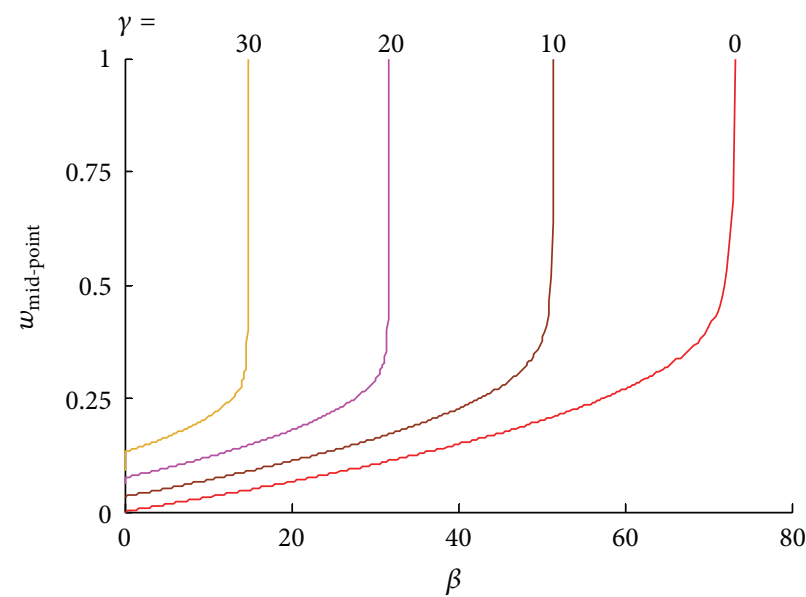

(d)

FIGURE 6: Effect of the Casimir force on the variation of nondimensional mid-point deflection versus the dimensionless pull-in voltage for nano/microplates with different aspect ratios: (a) $\alpha=1$, (b) $\alpha=0.75$, (c) $\alpha=0.5$, and (d) $\alpha=0.25$.

pull-in instability) is $41.61 \%$ of the initial gap counterpart. To compare the results of the present iterative approach with those of FE simulation, variation of the mid-point deflection versus the applied voltage is depicted in Figure 5. According to the results shown in Figure 5, there exists an excellent agreement between the findings of present iterative approach and those of 3D FE analysis carried out by COMSOL.

6.2. Effect of the Casimir Force. By decreasing the dimensions of electromechanical systems from microscale to nanoscale, the effect of dispersion forces may be considerable. To investigate the effect of Casimir force on the stable and unstable responses of the system, variations of the nondimensional mid-point deflection versus the dimensionless applied voltage for microplates with some different aspect ratios are depicted in Figure 6. According to this figure, by increasing the values of Casimir parameter, the mid-point deflection is increased which results in earlier pull-in instability of the system. The values of nondimensional pull-in parameter (i.e., $\beta_{\mathrm{PI}}$ ) versus the Casimir parameter of the system (i.e., $\gamma$ ) at some different aspect ratios of the plate are also given in
TABLE 3: Nondimensional pull-in voltage versus the Casimir parameter.

\begin{tabular}{lcccc}
\hline$\gamma$ & $\beta_{\mathrm{Pl}}(\alpha=1)$ & $\beta_{\mathrm{Pl}}(\alpha=0.75)$ & $\beta_{\mathrm{Pl}}(\alpha=0.5)$ & $\beta_{\mathrm{Pl}}(\alpha=0.25)$ \\
\hline 0 & 176.7 & 113.7 & 84.6 & 73.2 \\
20 & 134.7 & 73.2 & 45.3 & 34.5 \\
40 & 97.5 & 38.4 & 12.3 & 0 \\
60 & 63 & 6.9 & 0 & 0 \\
80 & 30.6 & 0 & 0 & 0 \\
100 & 0 & 0 & 0 & 0 \\
\hline
\end{tabular}

Table 3. As it is seen from this table, decreasing the aspect ratio (i.e., $\alpha$ ) reduces the nondimensional parameter of pullin voltage and the percentage of the reduction increases as the value of $\gamma$ is increased. Furthermore, according to Table 3, the system may be collapsed without applying the external voltage, if the Casimir parameter of the system reaches its critical value. It is to be noted that this condition is undesirable in designing N/MEMS and should be avoided. 


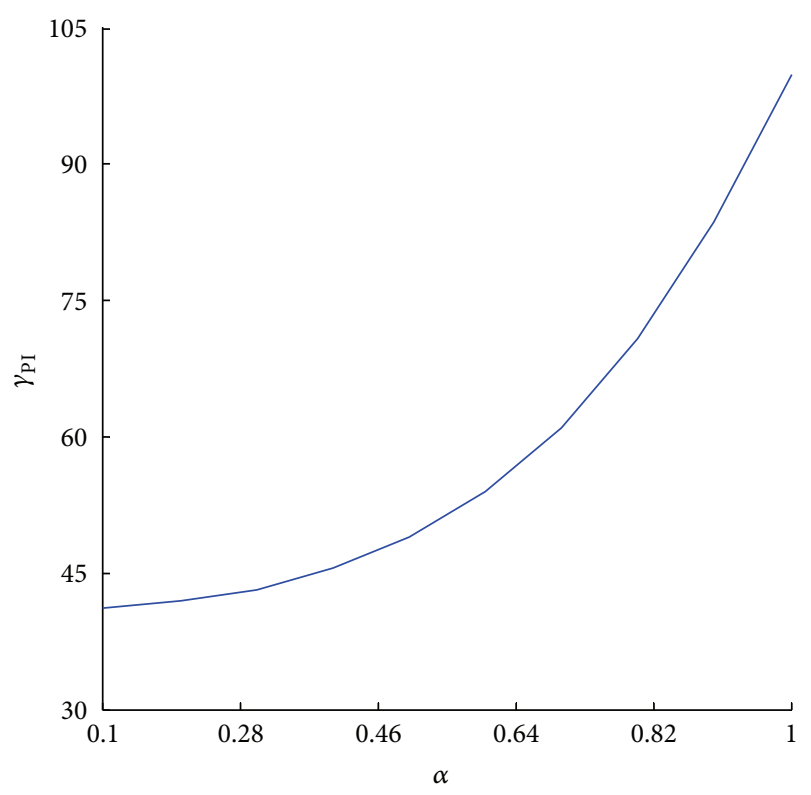

Figure 7: Variation of the critical Casimir parameter versus the plate's aspect ratio.

The variation of critical Casimir parameter versus the variation of the plate's aspect ratio is depicted in Figure 7. According to the results of this figure, the critical value of Casimir parameter increases with an increase of the plate's aspect ratio. According to (8), $\gamma$ is a function of geometrical and material properties of the system such as the initial gap between the two electrodes and the dimensions of the movable electrode. Based on this equation, when the initial gap between two electrodes is less than its minimum allowable size or the dimensions of the nano/microplate become greater than their allowable sizes, $\gamma$ reaches its critical value. In such conditions, the movable electrode collapses without applying the external voltage. Hence, such a system cannot work appropriately. The maximum allowable dimensions of the movable electrode as well as the minimum allowable initial gap can be easily determined using (8) as

$$
\begin{gathered}
a_{\max }=\sqrt[4]{\frac{240 D G^{5} \gamma_{\mathrm{cr}}}{\pi^{2} \hbar c}}, \quad b_{\max }=\alpha a, \\
G_{\min }=\sqrt[5]{\frac{a^{4} \pi^{2} \hbar c}{240 D \gamma_{\mathrm{cr}}}} .
\end{gathered}
$$

It is noteworthy that the initial gap between the two electrodes and the dimensions of the movable electrode should be controlled to avoid the collapse condition in N/MEMS.

\section{Conclusions}

The main idea of this study was to present an accurate novel method to investigate the behaviour of the parallel-plate nano/microcapacitors. To this end, the extended Kantorovich method tighter with the iterative procedure proposed by Tahani and Askari was used for obtaining both stable and unstable behaviors of the system. The results were compared and validated by previous theoretical and experimental findings as well as those of FE simulation carried out by COMSOL Multiphysics software. It was found that accounting for the Casimir effect decreases the pull-in voltage of the system especially for the cases with large Casimir parameter. Also, it was observed that the movable electrode may be collapsed without applying the external voltage for systems in which the Casimir parameter reaches its critical value. The latest finding was also utilized to obtain the critical dimensions of the movable electrode as well as the minimum allowable initial gap of the system.

\section{Conflict of Interests}

The authors declare that there is no conflict of interests regarding the publication of this paper.

\section{References}

[1] S. Srivastav, P. Bhardwaj, and Summit, "Fabrication, sensing \& application of MEMS/NEMS technology," International Journal of Computational Engineering \& Management, vol. 12, pp. 57-60, 2011.

[2] A. R. Askari and M. Tahani, "Investigating nonlinear vibration of a fully clamped nanobeam in presence of the van der waals attraction," Applied Mechanics and Materials, vol. 226-228, pp. 181-185, 2012.

[3] A. Nisar, N. Afzulpurkar, B. Mahaisavariya, and A. Tuantranont, "MEMS-based micropumps in drug delivery and biomedical applications," Sensors and Actuators B: Chemical, vol. 130, no. 2, pp. 917-942, 2008.

[4] M. Tahani and A. R. Askari, "Accurate electrostatic and van der Waals pull-in prediction for fully clamped nano/micro-beams using linear universal graphs of pull-in instability," Physica E, vol. 63, pp. 151-159, 2014.

[5] S.-A. Zhou, "On forces in microelectromechanical systems," International Journal of Engineering Science, vol. 41, no. 3-5, pp. 313-335, 2003.

[6] A. Gusso and G. J. Delben, "Influence of the Casimir force on the pull-in parameters of silicon based electrostatic torsional actuators," Sensors and Actuators A: Physical, vol. 135, no. 2, pp. 792-800, 2007.

[7] F. M. Serry, D. Walliser, and G. J. Maclay, "The role of the Casimir effect in the static deflection and stiction of membrane strips in microelectromechanical systems (MEMS)," Journal of Applied Physics, vol. 84, no. 5, pp. 2501-2506, 1998.

[8] H. Moeenfard, A. Darvishian, and M. T. Ahmaidan, "Static behavior of nano/micromirrors under the effect of Casimir force, an analytical approach," Journal of Mechanical Science and Technology, vol. 26, no. 2, pp. 537-543, 2012.

[9] C. Farina, “The Casimir effect: some aspects," Brazilian Journal of Physics, vol. 36, pp. 1137-1149, 2006.

[10] Y. T. Beni, A. Koochi, and M. Abadyan, "Theoretical study of the effect of Casimir force, elastic boundary conditions and size dependency on the pull-in instability of beam-type NEMS," Physica E: Low-Dimensional Systems and Nanostructures, vol. 43, no. 4, pp. 979-988, 2011.

[11] Y. T. Beni and M. Abadyan, "Use of strain gradient theory for modeling the size-dependent pull-in of rotational nano-mirror 
in the presence of molecular force," International Journal of Modern Physics B, vol. 27, no. 18, Article ID 1350083, 18 pages, 2013.

[12] Y. T. Beni, A. Koochi, and M. Abadyan, "Using modified couple stress theory for modeling the size-dependent pull-in instability of torsional nano-mirror under Casimir force," International Journal of Optomechatronics, vol. 8, no. 1, pp. 47-71, 2014.

[13] Y. T. Beni, A. R. Vahdati, and M. Abadyan, "Using ALE-FEM to simulate the instability of beam-type nano-actuator in the present of electrostatic field and dispersion forces, Iran," Iranian Journal of Science and Technology, vol. 37, pp. 1-9, 2013.

[14] W. H. Lin and Y. P. Zhao, "Dynamic behavior of nanoscale electrostatic actuators with Casimir force," Chaos, Solitons \& Fractals, vol. 23, pp. 1777-1785, 2004.

[15] A. Ramezani, A. Alasty, and J. Akbari, "Closed-form solutions of the pull-in instability in nano-cantilevers under electrostatic and intermolecular surface forces," International Journal of Solids and Structures, vol. 44, no. 14-15, pp. 4925-4941, 2007.

[16] A. Koochi, A. Kazemi, F. Khandani, and M. Abadyan, "Influence of surface effects on size-dependent instability of nanoactuators in the presence of quantum vacuum fluctuations," Physica Scripta, vol. 85, no. 3, Article ID 035804, 2012.

[17] Y. T. Beni, A. Koochi, A. S. Kazemi, and M. Abadyan, "Modeling the influence of surface effect and molecular force on pull-in voltage of rotational nano-micro mirror using 2-DOF model," Canadian Journal of Physics, vol. 90, no. 10, pp. 963-974, 2012.

[18] Y. T. Beni, M. Abadyan, and A. Koochi, "Effect of the Casimir attraction on the torsion/bending coupled instability of electrostatic nano-actuators," Physica Scripta, vol. 84, no. 6, Article ID 065801, 2011.

[19] A. Farrokhabadi, N. Abadian, R. Rach, and M. Abadyan, "Theoretical modeling of the Casimir force-induced instability in freestanding nanowires with circular cross-section," Physica E, vol. 63, pp. 67-80, 2014.

[20] X. Zhao, E. M. Abdel-Rahman, and A. H. Nayfeh, "A reducedorder model for electrically actuated microplates," Journal of Micromechanics and Microengineering, vol. 14, no. 7, pp. 900906, 2004.

[21] R. C. Batra, M. Porfiri, and D. Spinello, "Review of modeling electrostatically actuated microelectromechanical systems," Smart Materials and Structures, vol. 16, no. 6, pp. R23-R31, 2007.

[22] R. C. Batra, M. Porfiri, and D. Spinello, "Reduced-order models for microelectromechanical rectangular and circular plates incorporating the Casimir force," International Journal of Solids and Structures, vol. 45, no. 11-12, pp. 3558-3583, 2008.

[23] S. Timoshenko and S. Woinowsky-Krieger, Theory of Plates and Shells, McGraw-Hill, New York, NY, USA, 1959.

[24] J. N. Reddy, Energy Principles and Variational Methods in Applied Mechanics, John Wiley \& Sons, New York, NY, USA, 2002.

[25] A. R. Askari and M. Tahani, "Analytical approximations to nonlinear vibration of a clamped nanobeam in presence of the Casimir force," International Journal of Aerospace and Lightweight Structures, vol. 2, no. 3, pp. 317-334, 2012.

[26] A. R. Askari and M. Tahani, "An alternative reduced order model for electrically actuated micro-beams under mechanical shock," Mechanics Research Communications, vol. 57, pp. 34-39, 2014.

[27] COMSOL Multiphysics 4.3b Software Corporation, "Pull-In Voltage for a Biased Resonator-3D," 2013.
[28] J. P. Arenas, "On the vibration analysis of rectangular clamped plates using the virtual work principle," Journal of Sound and Vibration, vol. 266, no. 4, pp. 912-918, 2003.

[29] O. Francais and I. Dufour, "Normalized abacus for the global behavior of the diaphragms: pneu matic, electrostatic, piezoelectric or electromagnetic actuation," Journal of Modeling and Simulation of Microsystems, vol. 12, pp. 149-160, 1999.

[30] P. M. Osterberg, Electrostatically actuated microelectromechanical test structures for material property measurement [Ph.D. thesis], Massachusetts Institute of Technology, Cambridge, Mass, USA, 1995. 

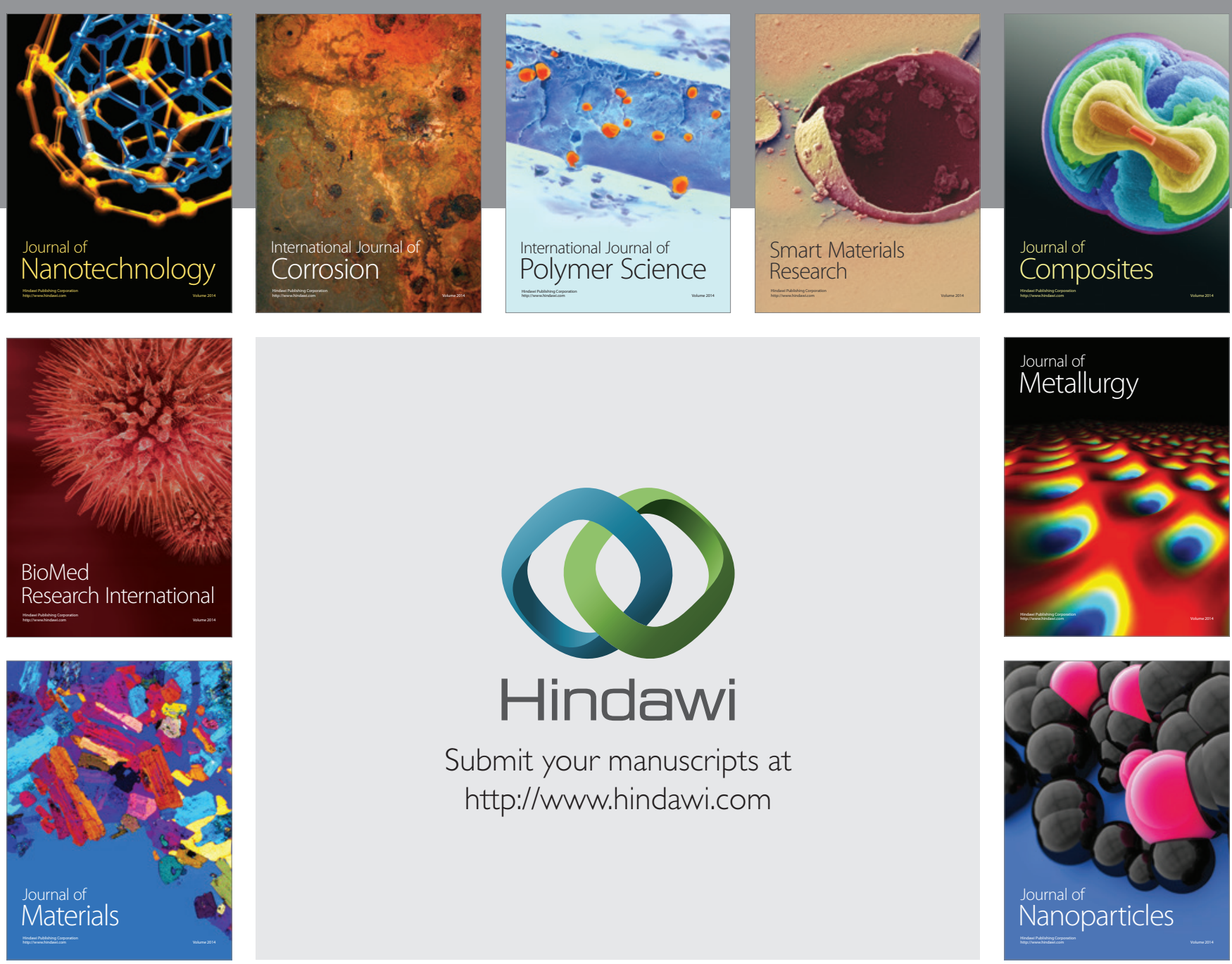

Submit your manuscripts at http://www.hindawi.com
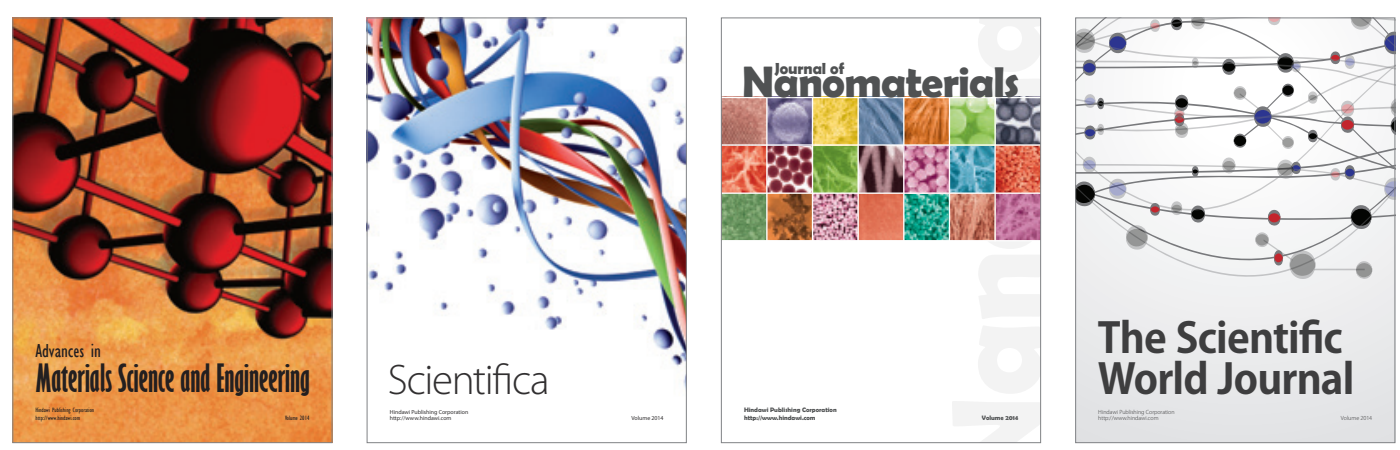

\section{The Scientific World Journal}
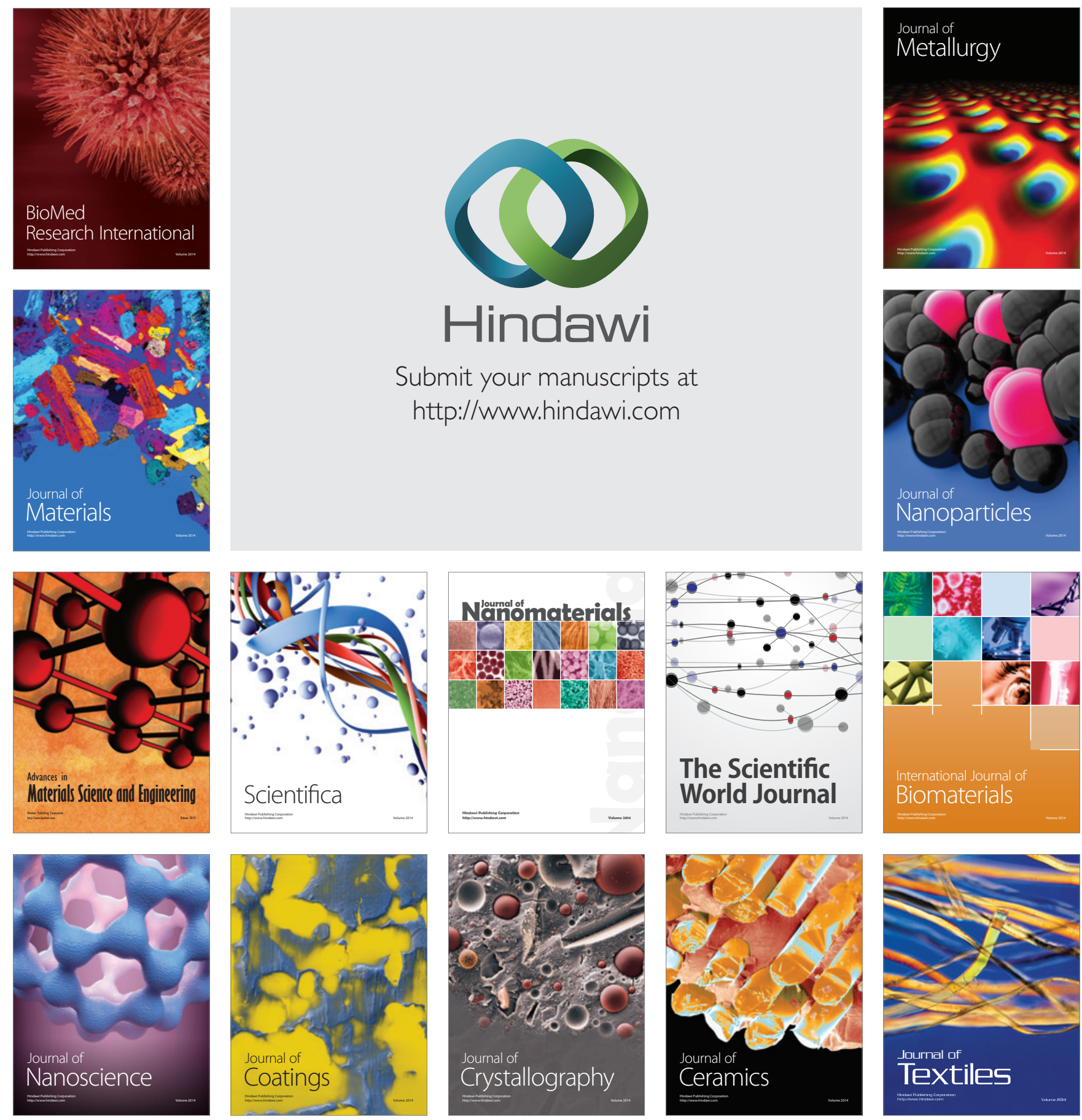